HoST - Journal of History of Science and Technology

Vol. 13, no. 2, December 2019, pp. 31-59

10.2478/host-2019-0012

\title{
The Silver Spring monkey controversy: changing cultures of care in twentieth-century laboratory animal research
}

\author{
Robert G. W. Kirk \\ Centre for the History of Science Technology and Medicine (CHSTM) \\ Faculty of Biology, Medicine and Health, University of Manchester
}

Robert.g.kirk@manchester.ac.uk

\begin{abstract}
In September 1981 police raided the Institute for Behavioral Research (Silver Spring, Maryland, USA) seizing a number of macaque monkeys in response to accusations of animal cruelty against the neuroscientist Edward Taub. Over the following decade a volatile battle was fought as Taub, the National Institutes of Health (NIH), and the nascent animal rights group People for the Ethical Treatment of Animals (PETA), contested the claims and decided the monkeys' fate. In spite of the monkeys having been surgically altered so as to be incapable of feeling pain a loose alliance of veterinarians, ethologists and animal advocates argued that they nonetheless suffered. Whilst this episode is often seen as a polarized confrontation between science and society, this paper argues that the Silver Spring monkey controversy saw two historically distinct cultures of laboratory animal care meet resulting in the development of new approaches to animal welfare in biomedical science.
\end{abstract}

Keywords: laboratory; animal; welfare; ethics; neuroscience 
On the 11th September 1981 police raided the laboratory of Edward Taub, a behavioural neuroscientist at the Institute for Behavioral Research (IBR) in Silver Spring, Maryland. Responding to accusations of animal cruelty, law enforcement officers removed seventeen macaque monkeys. A prolonged confrontation followed, fought in private and public arenas for over a decade, transforming seventeen hitherto unknown laboratory animals into household names: the "Silver Spring Monkeys." These events were a pivotal moment in the birth of the modern animal rights movement. ${ }^{1}$ The raid was orchestrated by a recently established animal advocacy group, the People for the Ethical Treatment of Animals (PETA), which subsequently grew to become a global force in animal rights. ${ }^{2}$ Less acknowledged, though equally significant, was the impact on Taub and scientific understanding of the brain. Taub never again worked with animals. Instead, he adapted what he had learned with monkeys to produce innovative new treatments for stroke induced illness in humans. This work was based on a radical new understanding of the brain's capacity for change now widely known as neuroplasticity evidence for which was a serendipitous outcome of the Silver Spring monkeys' unexpected longevity caused by their removal from the laboratory and subsequent legal limbo. Most importantly, the decade-long debate over the Silver Spring monkeys transformed public discourse on animal research and contributed to significant reform of the regulatory framework in the USA.

The Silver Spring monkeys are conventionally understood to represent the enduring conflict between the values of science and society, human need and animal rights. Without disputing this interpretation, or the significance of the Silver Springs monkeys for revitalizing the American animal advocacy movement, this article proposes a less polarised reading. It resists an explanatory frame based on societal demand for the imposition of animal care in its absence. Instead, this account explores how conflicting approaches to caring for and understanding the welfare of laboratory animals lay at the heart of the controversy. Older approaches to animal care conflicted with and were eventually superseded by newer practices grounded in considerably wider matters of concern. Bringing these interactions to the fore reveals the historically situated and complex ways in which cultural, societal and scientific values interact to drive change within the public perception, practice and governance of animal research.

The Silver Spring monkey controversy was a moment where two historically distinctive regimes of valuing and caring for laboratory animals conflicted. We might think of these "regimes" as distinct examples of cultures of care. In recent years, theorizing "care" has become a focal point in the sociological study of science. Friese's characterization of "care as science"

\footnotetext{
${ }^{1}$ Lawrence Finsen, The Animal Rights Movement in America, from compassion to respect (New York: Twayne, 1994).

2 PETA was established in March 1980 by Ingrid Newkirk and Alex Pacheco.
} 
is one example of a growing interest in the analysis of care. ${ }^{3}$ Here, Friese shows how animal care has become linked to the successful "translation" of animal research to human benefits within contemporary biomedical science. ${ }^{4}$ Characteristic of recent theorizations of care is the move away from understanding care as a form of normative ethics. Instead, care is shown to be situated, multiple and emergent in the materialized doing of science. For Puig de la Bellacasa care is dependent on a relational ecology. It requires "knowledge and curiosity regarding the needs of an 'other' - human or not - and these become possible through relating, through refusing objectification". Human and animal become mutually constitutive of the other through situated relational processes as care "inevitably transforms the entangled beings." For historians, these theorized accounts of care are useful because to be situated is to be historically constituted. Accordingly, care can only be properly understood within its historical context. As a concept, care can thereby provide an analytic bridge between the historical and sociological study of animal research. ${ }^{6}$

The two conflicting cultures of care at the centre of the Silver Spring monkey controversy are products of different though closely related periods of time. The first and historically earlier culture of care, dominant from the early twentieth century through to the 1960s, framed care as an instrumental tool subservient to the needs of the experimental system. The lead scientist was usually responsible for animal care and often managed their own animal house and laboratory (as opposed to centralized shared facilities). The first part of this article describes how Taub came to embody this ethos. Early in his career, Taub trained as a behaviourist displaying little interest in the internal mental lives of animals. Monkeys were surgically transformed into highly specialized biomedicalized bodies. Animal care was understood in relation to biological health and absence of physiological pain. However, as Taub's research developed, the canvas of concerns shaping animal care broadened. Taub gradually recognised the epistemological importance of his monkeys' emotional and affective states. He gradually moved away from radical behaviourism which in turn expanded the boundaries of his culture of care. Reconstructing this process reveals how Taub's experimental system generated specific forms of curiosity about laboratory monkeys that gave shape to situated care practices.

\footnotetext{
${ }^{3}$ Gail Davies, "Caring for the Multiple and the Multitude: Assembling Animal Welfare and Enabling Ethical Critique," Environment and Planning D: Society and Space 30, no. 4 (2012): 623-38; Tone Druglitrø, "Skilled Care' and the Making of Good Science," Science, Technology and Human Values 43, no. 4 (2017): 649-70; Beth Greenhough and Emma Roe, "Ethics, Space, and Somatic Sensibilities: Comparing Relationships between Scientific Researchers and Their Human and Animal Experimental Subjects," Environment and Planning D: Society and Space 29, no. 1 (2011): 47-66.

${ }^{4}$ Carrie Friese, "Realizing Potential in Translational Medicine: The Uncanny Emergence of Care as Science," Current Anthropology 54, no. S7 (2012): S129-S138.

${ }_{5}^{5}$ Maria Puig de la Bellacasa, Matters of Care Speculative ethics in more than human worlds (Minneapolis: University of Minnesota Press, 2017), 90.

${ }^{6}$ Relating these two fields is an enduring concern; e.g. Peter Dear and Sheila Jasanoff, "Dismantling Boundaries in Science and Technology Studies," Isis 101, no. 4 (2010): 759-774.
} 
However, throughout, new matters of concern emerged only in relation to and as an effect of experimental need and the trajectory of scientific research.

The second part of the article moves to the events following the 1981 raid. It explores how the seizure of the Silver Spring Monkeys exposed Taub's working practices to an emergent culture of care which presumed a much broader canvass for understanding the needs of laboratory animals. Within this regime, animal care was a specialised and diversified body of expertise. Responsibility was dispersed amongst an eclectic specialist community dedicated to animal care where veterinary expertise was particularly predominant. ${ }^{7}$ Within this broader approach to laboratory animal care new forms of knowledge were considered viable tools for shaping laboratory animal care. This included veterinary medicine, zoology, ethology, evolutionary theory and anthropomorphic reasoning. This alternate culture of care shared many commonalities with Taub's yet encompassed very different reference points. For instance, both incorporated a concern for the social and psychological wellbeing of animals. However, the former assumed animal welfare was a concern in and of itself understood through reference to animal species and behaviour in nature. Consequently the species specific needs of animals in the wild could inform the needs of animals in the laboratory. Whereas Taub believed his laboratory animals to be unique beings with highly specialist needs comprehensible only through reference to the experimental system. For Taub, "nature" was indirectly if at all relevant to provision of care in the laboratory. Taub's culture of care began with the experimental system and worked outward allowing matters of concern to emerge as they were found to have epistemological relevance. The differences between these two approach to a culture of care were revealed in the courts, congressional hearings, the institutional deliberations of the National Institutes of Health, media coverage and within wider public discourse, following the 1981 seizure of the silver spring monkeys. Taub's approach was challenged and eventually superseded by a culture of care which gave much more importance to the perceived needs of the animal as understood through reference to behavioural needs in nature. In this way the legacy of the Silver Spring monkey controversy was the broadening of matters of concern which made up an acceptable culture of care within animal research laboratories. ${ }^{8}$

\section{Care as a moral economy of science?}

Edward Taub arrived at Columbia University (NY) in 1956 driven by a fascination with experimental psychology and the then in vogue behaviourism. William "Nat" Schoenfeld

\footnotetext{
${ }^{7}$ Larry Carbone, What animals want. Expertise and advocacy in laboratory animal welfare policy (New York: Oxford University Press, 2004).

${ }^{8}$ For a comparable study in the Danish context see Lene Koch and Mette N. Svendsen, "Negotiating Moral Value: A Story of Danish Research Monkeys and Their Humans," Science, Technology and Human Values 40, no. 3 (2015): 368-88.
} 
and Fred Simmons Keller had established the first undergraduate course in behaviourism at Columbia in in 1947. ${ }^{9}$ Here, Taub learnt sophisticated experimental techniques such as operant conditioning (the modification of an organism's behaviour through positive and negative reinforcement which presupposed behaviour to be a reflex response to a stimulus). He also absorbed the behaviourist refusal to consider internal motivational states such as will, emotion or mind as adequate scientific explanations for behaviour. ${ }^{10}$ In 1957 , seeking opportunities to practice experimental science, Taub took a position working with the neurosurgeon, A. J. Berman, at the Isaac Albert Research Institute of the Jewish Chronic Disease Hospital (Brooklyn). ${ }^{11}$ Taub gave little thought to the possible consequences of entangling different disciplinary worlds. Academically, he was learning and perhaps more importantly he had found a way to put what he was learning into practice.

Combining the conceptual and experimental approaches of behaviourism with the disciplinary knowledge and surgical techniques of neurophysiology provided Taub with a unique experience. Much later, Taub's skillset would become recognisable as a form of behavioural neuroscience. In the 1950s, however, this field was yet to formalise. Taub's modest yet original doctoral project capitalised on this background by seeking to confirm "Sherringtonian reflexology" using new experimental approaches derived from behaviourism. Originating in the work of Charles Sherrington and F. W. Mott in late nineteenth century Britain, Sherringtonian reflexology had become a central tenant of neurophysiology. In carefully designed experiments Sherrington and Mott surgically severed the sensory nerves in a rhesus monkey limb causing the animal to lose the sense of touch yet retain motor nerves. This technique, subsequently known as deafferentation, required a very high level of surgical skill. ${ }^{12}$ This work showed that deafferented animals made no effort to move a desensitized limb in spite retaining their physiological capacity to do so. Without sensory experience, animals were unable to move. Thus "Sherringtonian reflexology" established sensation as a necessary component of voluntary movement.

Although behaviourism had little direct interest in neurophysiology its conceptual framework

\footnotetext{
9 James A. Dinsmoor, "Keller and Schoenfeld's principles of psychology," The Behaviour Analyst 12, no. 2 (1989): 213-19.

${ }^{10}$ John A. Mills, Control. A history of behavioural psychology (New York: New York University Press, 1998).

11 “Curriculum Vitae,” Box 58, folder “Taub, Edward CV 1983.” Neal E. Miller Papers. Manuscripts and Archives, Yale University Library, hereafter NEM.

${ }^{12}$ Frederick Walker Mott and Charles Scott Sherrington, "Experiments upon the influence of sensory neurons upon movement and nutrition of limbs. Preliminary communication," Proceedings of the Royal Society of London 57, no. 340-346 (1895): 481-488. Working at the height of the late nineteenthcentury antivivisectionist controversy, Mott and Sherrington emphasised that in "all our operations the animals have been deeply anaesthetised with chloroform and ether" (481).
} 
had absorbed the language of reflexology. ${ }^{13}$ Reflexology explained behaviour in terms of a response to a physical environmental factor (i.e. stimulating a sensory nerve triggered a motor nerve eliciting a response). As an explanatory device, reflexology allowed behaviourist to account for complex behaviour without presupposing ephemeral internal states such as will or mind. Accordingly, Taub's proposal to apply behaviourist techniques to confirm Sherringtonian reflexology was far from controversial (even if his ability to do so was at the time quite unique). Whilst replication would be no more than a footnote within neurophysiology, association with a well-established neurophysiological principle would provide behaviorism significant credibility as an experimental science. In any case, the doctoral project was primarily to establish Taub's capacity to design and conduct novel experimental research. Confirmation of Sherringtonian reflexology would in turn confirm Taub's ability as a scientist. It was, therefore, something of a blow that Taub's data departed wildly from expectation.

Using standard behaviourist techniques Taub began by conditioning monkeys to avoid an electric shock by flexing a forelimb in response to an auditory stimulus. He then subjected the animals to deafferentation. Successful deafferentation was a highly skilful surgical technique which Taub patiently learned at the Isaac Albert Research Institute. It required the meticulous severing of sensory nerves in a limb so as to remove the capacity for somatic feeling whilst leaving motor nerves intact. Following deafferentation, Taub expected each animal to fail to move their arm in response to the auditory stimulus thereby confirming Sherringtonian reflexology. Initially, all went to plan. During a recovery period following surgery each monkey's deafferented limb hung loosely and unused. Only the normal limb was employed to aid movement, climbing and eating just as Sherrington and Mott had described. However, drawing on the behaviourist commitment to the absolute removal of mind from an experimental scenario, Taub introduced an improvement to the original experimental design to rule out the possibility than an animal "chose" not to use the deafferented arm. $\mathrm{He}$ introduced a physical restraint on the monkey's normal forelimb to prevent its use during the conditioning experiment. Taub was shocked to see monkeys move their deafferented arm with uniform consistency once their "normal" limb was restrained. ${ }^{14}$ Something was wrong, yet Taub's experimental design proved robust. The data was conclusive. By applying behaviourist techniques to a neurophysiological problem Taub had inadvertently disproved rather than confirmed Sherringtonian reflexology.

Rather than welcoming Taub's corrective neurophysiologists and behaviourists rose to defend

\footnotetext{
${ }^{13}$ At Columbia, behaviourist research and teaching was grounded in the language of reflex. See Fred S. Keller and Willian N. Schoenfeld, "Psychology and the Reflex" in Principles of Psychology: A systematic text in the science of behavior, Fred S. Keller and Willian N. Schoenfeld, 1-14 (New York: AppletonCentury-Crofts Inc., 1950).

${ }^{14}$ H. D. Knapp, E. Taub E, and A. J. Berman, "Effect of deafferentation on a conditioned avoidance response," Science 128, no. 3328 (1958): 842-3.
} 
Sherringtonian reflexology from what each saw as an unqualified interloper in their field. Schoenfeld, now serving as Taub's doctoral supervisor, was vociferous in his objection. He attended Taub's thesis defence in a highly emotional state to refute the work of his own student. Later, Schoenfeld blocked the award of Taub's PhD by failing him on a basic taught course. ${ }^{15}$ In spite of these setbacks Taub retained a faith that the accumulation of more experimental data would eventually demonstrate the need to revise Sherringtonian reflexology. At the Isaac Albert Research Institute, Berman diligently acknowledged Taub's contribution to their research programme regardless of the absence of a $\mathrm{PhD}$. By naming Taub as co-investigator on several successful grant applications, Berman helped Taub build an impressive track record underpinned by substantial research funding from the National Institutes of Health (NIH) extramural programmes. This allowed Taub and his collaborators to systematically refute criticisms of their work whilst strengthening the empirical evidence for the reality of purposive movement without somatic sensation during the 1960s. Patiently, Taub chipped away at Sherringtonian reflexology and its defenders. In a series of meticulously constructed papers Taub ruled out the popular criticism that that vision was in some way compensating for sensory input. He also showed that deafferented limbs could be used where behavioural conditioning occurred after surgery. ${ }^{16}$ On the basis of nearly a decade and a half's experimental research and having transferred to New York University Taub was awarded a PhD in 1969.

At the same time, Taub's unconventional work was beginning to be associated with a new and equally unconventional understanding of the brain known as neuroplasticity. This held that the brain could adapt its functions in the course of an organism's life (for instance in response to injury). Neuroplasticity was controversial and widely dismissed in favour of the long established belief that the adult brain was fixed. An extensive 1967 review sceptically suggested that "inadequate technique rather than 'plasticity' of the brain" may account for recent criticisms of the fixity of brain function. ${ }^{17}$ Taub had not yet explicitly located his work within "neuroplasticity" as he did not believe he had conclusively demonstrated such a phenomenon. Nevertheless, the direction of travel implicit to his work was clear to others. In 1968, Taub published an overview of his research framed as a defence but also a fundamental critique of the assumption that sensory information was required for movement. In addition to

\footnotetext{
15 Taub had not completed the final exam for Schoenfeld's course. Normal procedure was to award a student an "incomplete" and enter a mark once the exam had been taken. Instead, Schoenfeld failed Taub due to his "insolence." Taub later recalled Schoenfeld appeared "very angry" following "a public debate on the relevance of my research to his theory ... he walked out." Quoted in Caroline Fraser, "The Raid at Silver Spring," New Yorker, April 19, 1993, 66-84, on 67.

${ }^{16}$ E. Taub, S. J. Ellman, and A. J. Berman "Deafferentation in Monkeys. Effect on Conditioned Grasp Response," Science 151, no. 3710 (1966): 593-594; E. Taub, R. C. Bacon, A. J. Berman, "Acquisition of a trace-conditioned avoidance response after deafferentation of responding limb," Journal of Comparative and Physiological Psychology 59, no. 2 (1965):275-79.

17 A. M. Laursen, "Higher functions of the central nervous system," Annual Review of Physiology 29, no. 1 (1967):543-572, on 564.
} 
refuting Sherringtonian reflexology, Taub extended his critique to the behaviourist exclusion of the mind from science. Adopting a more combative tone, Taub argued that the behaviourist commitment to Sherringtonian reflexology was no more than "a sort of glue to hold a number of learning theories together on what appeared to be empirical grounds, in the face of apparently contradictory evidence." 18 This was a disingenuous commitment designed to circumvent an appeal to mind. In contrast, Taub suggested that movement without somatic input was:

clearly a type of thinking by any worthwhile definition ... deafferentation research offers one type of window into the processes by virtue of which the CNS [central nervous system] achieves autonomy; by virtue of which the organism thinks. ${ }^{19}$

Taub was now tearing down a fundamental philosophical tenet of behaviourism. In concluding that "the concept of self-produced or voluntary movement must now be exhumed and re-examined experimentally" he reintroduced internal motivation as a legitimate object of inquiry for the behavioural sciences. ${ }^{20}$

Acknowledging nonhuman sentience did not, however, introduce new moral values or ethical practices. Monkeys had emerged as thinking beings within Taub's experimental system as a consequence of his experimental epistemology. For Taub, the value of deafferented monkeys lay in their function within the experimental system. However, this does not mean that their welfare was not a concern. On the contrary, the health and welfare of deafferented monkeys was critically important. Taub's monkeys were created through substantial investment of time, money and high levels of surgical skill. Each monkey was individually cared for in large part due to their being a unique form of life designed to fulfil specific experimental functions within the laboratory. As unique partly manufactured forms of life, deafferented monkeys required highly specialised forms of care which evolved in relation to the experimental system. When Taub began a new line of inquiry in the 1970s, for instance, seeking to understand whether sensation was necessary for a developing organism to learn movement, the new experimental system allowed new matters of care to emerge. Hitherto, Taub had worked with adult animals. Now, he deafferented monkeys on birth prior to any life experience. By 1975, Taub had perfected highly specialized foetal neurosurgery techniques to conduct deafferentation in the womb prior to birth. Creating animals that had never experienced somatic sensation yet developed motor capacity equal to "normal" animals by the age of 3 months undermined any

\footnotetext{
${ }^{18}$ E. Taub, "Movement in nonhuman primates deprived of somatosensory feedback," Exercise and sport sciences reviews 4, no. 1 (1976): 335-74, on 359.

19 Taub, "Movement in nonhuman primates," 359.

20 E Taub and A. J. Berman, "Movement and learning in the absence at sensory feedback," in The Neuropsychology of spatially orientated behaviour, ed. S. J. Freedman, 173-92 (Homewood, Illinois: Dorsey Press, 1968), 189-90.
} 
claim that sensation was a necessary component of movement.

Foetal surgery had a high level of mortality with over half of the animals dying. ${ }^{21}$ Those monkeys that survived had heightened value as research objects. Once subjected to long periods of training and conditioning, their value was enhanced even further as they became integral parts of an experimental system that would be difficult and costly to replace. The unique bodily and behavioural characteristics which made the monkeys integral to the Taub's experimental system helped constituted increased concern for their care and welfare. Neurosurgery, for instance, required the prevention of head movement with the standard method being restraint by means of screws surgically implanted in the skull. ${ }^{22}$ However, restraining screws posed a serious post-operative threat of infection that could undermine the long-range studies which Taub intended. Consequently, surgical interventions were minimized as they placed the animal at a risk "more than is desired for animals made especially valuable by either long training or successful recovery from life-endangering procedures." ${ }^{23}$ Taub worked to develop nonsurgical methods of head restraint. An initial possibility was the construction of individualised tight fitting helmets but this was abandoned in favour of a mechanical restrain system that held the head in place. In examples such as this, where Taub worked to improve his experimental techniques so as to minimize the risk they posed to a monkey's health and welfare, we can see the dynamic relationship between experimental practice and animal care. The overriding ethos was instrumental. The reference points for this culture of care evolved from the dynamic interplay of changing experimental needs and those of the monkey's welfare. The ultimate goal being to ensure the experimental system worked.

Similar interplay can be found throughout Taub's animal care and husbandry practices. As well as extending his research programme further back into the life of an animal, Taub also expanded his deafferentation investigations across the monkey's body. Under Sherringtonian reflexology there had been no reason to apply deafferentation to more than one limb. However, if desensitised limbs could be used new research questions followed such as how an animal might respond to loss of sensation in multiple limbs? Building out from "simple" deafferentation of a single limb to bilateral deafferentation of multiple limbs constituted a new experimental programme that introduced a range of new challenges requiring new approaches to animal care. Post-surgical recovery times, for example, were significantly lengthened as during the first two weeks following bilateral deafferentation a monkey's limbs were virtually useless. On average

${ }^{21}$ Taub, "Movement in nonhuman primates," 359.

${ }^{22}$ G. C. Sheatz, "Electrode holders in chronic preparations: A. Multilead techniques for large and small animals," in Electrical stimulation of the brain, ed. D. E. Sheer, 45-50 (Austin, TX: University of Texas Press, 1961).

${ }^{23}$ I. A. Goldberg, E. Kowler, and E Taub, "A nonsurgical method for restraining the head during behavioral tests in primates," Behavioral Research Methods and Instruments 5, no. 3 (1973): 309-10, on 309. 
it took up to six months for full movement to return. ${ }^{24}$ During this period monkeys required "[e]xtensive nursing care ... frequently including daily bandaging and passive exercise." As movement returned the animals required more not less care because:

[d] eafferented monkeys have a tendency to sustain severe damage to their affected extremities, frequently as the result of self-mutilation and sometimes simply because the lack of sensation eliminates a warning system signalling when the danger of injury is imminent. ${ }^{25}$

One of the most common acts of self-mutilation was for a monkey to chew digits on deafferented limbs. This risked infection but equally undermined their ability to complete standardized tests of precision movement. ${ }^{26}$ Here again, animal welfare concerns were entangled with the needs of the experimental system. Care served the needs of science as much as those of the animal because the:

extent to which an individual animal exhibits movements following deafferentation will, of course, depend on the degree to which its limbs have escaped injury. To estimate maximal function, it is necessary to observe only those animals with essentially undamaged extremities. ${ }^{27}$

Another example is found in the tendency of deafferented animals to be far more sensitive to unseen and unexpected changes in the environment. Consequently, greater attention was applied to shaping their living space and their emotional wellbeing. Taub explained that when:

a deafferented animal is calm and ambulating slowly its movements may sometimes approximate those of a normal animal. However, when the animal becomes excited, as in trying to escape from an experimenter, the coordination tends to become degraded. ${ }^{28}$

In this way affective states emerged as matters of care. Managing the entanglement of animal welfare, animal emotion, experimental need and the relationship between human and monkey in the experimental encounter was at the heart of Taub's culture of care. As such, we might think of Taub's practice as an example of a moral economy of science.

Borrowed from the social historian E. P. Thomson, the proposition of a moral economy of science has been variously developed for different ends within the history of science. The concept is generally invoked to chart the situated historically contingent social factors which

\footnotetext{
${ }^{24}$ Taub and Berman, "Movement and learning."

25 Taub, "Movement in nonhuman primates," 343.

26 Tests required picking up raisins between thumb and forefinger from the shallow wells of a dexterity board. Adapted from J. Cole, "Three tests for the study of motor and sensory abilities in monkeys," Journal of Comparative and Physiological Psychology 45, no. 3 (1952): 226-230.

27 Taub, "Movement in nonhuman primates," 343.

${ }^{28}$ Ibid.
} 
shape scientific practice whilst simultaneously tracing the conservative features which regulate and systematize scientific communities. ${ }^{29}$ However, Lorraine Daston has proposed a difference sense of moral economy. For Daston, the moral economy of science encompasses the "web of affect-saturated values that stand and function in well-defined relationship to one another." ${ }^{30}$ This includes the subjective and affective elements which are integral to scientific epistemology at a certain time and place but are not explicitly recognised as such. By emphasising the central role of emotional and affective experience in the production of scientific knowledge, Daston's concept of the moral economy of science allows the consideration of care within historical accounts of the epistemology of experimental science.

Accordingly, the way in which Taub managed the complex interrelationships of epistemological and welfare concerns, manifest in his scientific and animal care practices, produced a situated culture of care bounded by the needs of the experimental system that can be understood as a Dastonian moral ecology of science. Its reference point was primarily epistemological: matters of concern became such when they were linked to experimental need. The emotional life of animals (an almost heretical subject for a behaviourist) emerged when Taub recognised that calm monkeys could ambulate near to normal without injuring themselves whereas an excited or agitated animal lost such control posing a danger to themselves (and the continuation of experimental work). This entangled process illustrates the situated, emergent and relational characteristic of care practice. Rather than a set of normative values embedded in ethical and regulatory practice, within the animal dependent experimental sciences care is highly situational - an emergent practice which co-develops over time in relations to other factors not least epistemological and experimental needs. One had to know each monkey intimately, how to approach them and how to engage so as to maintain their sense of calm. Each monkey was to this extent an individual but also part of a collective, a co-contributor to their own experience of life in the laboratory. For Haraway, embodied care practices of "becoming with" such as these are equally forms of "becoming worldly"; processes that recognise relating with others to be transformative acts of constructive world building. ${ }^{31}$ In a similar way, for Taub experimental system and monkey were mutually constituted in the doing of his research. Accordingly, Taub's culture of care was bounded by the experimental system. Taub firmly believed that his monkeys were a laboratory product of experimental science. So much so he failed to see how knowledge derived from the same species in a "natural" setting could be relevant to their care and welfare.

\footnotetext{
${ }^{29}$ Kohler used the term to capture the customs, obligations and implicit expectations that governed the development of early drosophila genetics, see Robert Kohler, Lords of the Fly, Drosophila Genetics and the Experimental Life (Chicago: University of Chicago Press, 1994). See also Janet Atkinson-Grosjean and Cory Fairley, "Moral ecologies in science: from ideal to pragmatic," Minerva 47, no. 2 (2009): 147-70; Bruno J Strasser, "The experimenter's museum: GenBank, natural history, and the moral economies of biomedicine," Isis 102, no. 1 (2011): 60-96.

${ }^{30}$ Lorraine Daston, “The Moral Economy of Science," Osiris 10 (1995): 2-24, on 4.

${ }^{31}$ Donna Haraway, When Species Meet (Minneapolis: University of Minnesota Press, 2007).
} 
Surgical interventions and a life in the laboratory had made Taub's monkeys so different to the wild species that the latter had no bearing on the welfare needs of the former. This was the principle difference between Taub's culture of care and that which gave shape to the Silver Spring monkey controversy in the 1980s.

\section{Care as a political ecology of science?}

In the late summer of 1981 Taub was on vacation having entrusted the care of his research monkeys to Alex Pacheco, a young student who had volunteered to work in Taub's laboratory earlier in the year. Pacheco had spoken passionately of his desire to build a career as a research scientist. However, he was actually an active animal rights campaigner and co-founder of the recently established People for the Ethical Treatment of Animals (PETA). In Taub's absence Pacheco invited five "experts" in animal welfare into the facility to assess the monkeys. Michael W. Fox, Donald Barnes, Geza Teleki, John McArdle and Ronnie Hawkin were carefully selected for their experience but also for their known sympathy for and connections to animal advocacy organisations. ${ }^{32}$ Each was asked to comment on the state of seventeen laboratory monkeys who subsequently became the "Silver Spring monkeys." Highly critical signed affidavits were prepared condemning the laboratory as "extremely filthy" and asserting that the "animals appeared unhealthy, and evidenced a lack of veterinary care." 33 When passed to the local law enforcement they triggered the police raid and removal of all seventeen monkeys accompanied by almost immediate media coverage. The speed at which informed and graphic media reports appeared suggests that the public imagination was purposefully targeted as part of a wider strategy to change socio-political thinking on animal research. Well briefed journalists described how County police "found monkeys that were in such physical and mental stress that the animals appeared to have bitten off their fingers and arms." ${ }^{34}$ In contrast, local and state agencies which may (or may not) have had a stake in what was a highly unprecedented situation were entirely unprepared. Prosecutors scrambled to respond within a context of growing confusion. People who worked with Taub were arrested, jailed and released as deputies and court officials tried to understand who ultimately was responsible for the monkeys and what if any crime had been committed. At one point, the monkeys themselves disappeared having been released into the care of representatives of a local animal

\footnotetext{
32 Barnes worked closely with the Animal Protection Institute of America, Fox was scientific director of the Humane Society of the United States, McArdle was an active member of the Scientists Group for Reform of Animal Experimentation, Teleki worked closely with a number of conservation groups including the Jane Goodall Institute.

33 The use of animals in medical research and testing. Hearings before the subcommittee on science, research and technology of the committee on science and technology U.S. House of Representatives, 97th congress October 13th, 14th, 1981 [no. 68], 82-3.

${ }^{34}$ Ronald White, "Monkeys seized in raid missing from Md. Home," The Washington Post,September 24, 1981, B1.
} 
welfare organisation. Taub spoke of his concern for the animals' welfare offering a reward for their return fearing that they "may be killed with the excuse that this was for their own good." 35 The animals were eventually returned when it became apparent that the monkeys constituted key evidence without which a prosecution was impossible.

Within the media and wider public discourse, the institutional deliberations of the NIH, the legal case and congressional hearings, Taub was accused of wanton cruelty and a failure to provide adequate care for his animals. PETA worked to amplify this accusation by depicting the monkeys as "victims" and the regulatory system as unfit for purpose. Graphic evidence was distributed which simultaneously humanised the animals (e.g. through attribution of personal names) whilst vividly describing their suffering. A monkey named "Sisyphus", for instance, was described as having been "forced to eat food contaminated with his own faecal matter and urine in order to survive." ${ }^{36}$ Taub disputed such claims believing not unreasonably that he was the victim of a sting. ${ }^{37}$ During his vacation the two staff employed to clean, maintain and care for the monkey colony rooms alongside Pacheco were absent for seven of the fourteen days. Over the preceding two years the same staff had been absent for only three days. This was never adequately explained though Taub noted that Pacheco's "observers" (he refused to acknowledge their "expertise") had each visited on one of the absentee days. When asked if Taub believed he had been set up Taub's response was that the statistical likelihood of the absentee record being "due to chance was only eight times in 10 billion" but "probability is proof for scientists, but not in a court of law." 38

Behind the language of cruelty lay a more nuanced procedural critique grounded in a culture of care quite different to the moral economy of science that Taub had developed during his thirty year career. The scientific, legal, institutional, and political arguments over the Silver Spring monkeys, conducted in the private and public domain, operated as a platform to advance a culture of care that valued the animal in and of itself. This perspective brought with it a much broader canvass of concerns raising new questions as to who had the appropriate expertise to interpret the health and welfare needs of the Silver Spring monkeys. Taub claimed that as the lead research scientist he and he alone had the expertise, experience and authority to understand and provide for the needs of what were highly specialised laboratory animals.

35 White, "Monkeys seized."

36 "PETA Facts about the victims" in Box 5 011, Folder 1, "Taub, Edward". Animal Welfare Institute Records, MC 00344, Special Collections Research Center, North Carolina State University Libraries, Raleigh, NC.

37 Taub had been previously targeted. In 1977 Fay Brisk, a vocal animal advocate who had played a key role in campaigning for the 1966 Animal Welfare Act, lodged a complaint with the NIH alleging inhumane conditions. A subsequent inspection found the monkey colony rooms to be "clean and adequate" whilst "animals appeared to be healthy and well cared for." See The use of animals, 103-104.

${ }^{38}$ Nancy Heneson, "Cruelty to animals: the state versus the scientist," New Scientist 92, no. 1282 (December 3, 1981): 672-674, on 673. 
Opposing this view was a broad collective of animal advocates, veterinarians and NIH officials who subscribed to what might be described as a humanitarian veterinary perspective. This latter culture of care placed value on bodily health as well as the social, psychological and holistic needs of animals. It encompassed a range of expertise including veterinary medicine, ethology, psychology and zoology. Most importantly, it took the experience of animals in wild and captive sites as the starting point for understanding animal welfare in captivity. Contrary to Taub, all five signatories of original affidavits prioritised the "natural" behaviour of animals over their artificial experience in the laboratory environment. Each had expertise and experience working with and meeting the needs of non-human primates but none had any specialist knowledge of deafferentation. Taub's defence rested on the unique status of the Silver Spring monkeys as animals that had evolved within an artificial experimental system and could only be understood in the context of deafferentation. Their physiological and behavioural needs were distinctive due to having been surgically altered at a young age (or even prior to birth). On these grounds, Taub rejected the relevance of the scientific credentials of his critics going so far as to discount veterinarians appointed by the NIH as lacking the experience to adequately care for the Silver Spring monkeys.

These different approaches to cultures of care in the laboratory were made visible when Taub stood trial before Judge Stanley Klavan between 27th October and 23rd November 1981. Taub was accused of 119 counts of animal cruelty. Graphic imagery and film was marshalled by Roger Galvin, prosecuting assistant state's attorney, displaying monkeys whose limbs bore multiple open and healed scars. Some lacerations were bandaged but several open wounds had no indication of dressings. According to the prosecution it was clear that the animals "suffered unnecessary pain." ${ }^{39}$ Noting the absence of a record of "regular visits ... necessary for vets to take a look at the animals" Galvin also asserted that the "monkeys received no veterinary care" likely to reduce the economic cost of proper care (though there was no evidence to support such a supposition). ${ }^{40}$ To the lay eye it was difficult to interpret these images as anything but neglect bordering on cruelty. Veterinary witnesses for the prosecution saw the same, adding in their expert testimony that the open wounds laced animals at a considerable risk of infection. The prosecution had no doubt that the monkeys had been deprived of veterinary care and as a result experienced considerable and unnecessary suffering.

Taub disputed all the accusations against him. What appeared to be cruelty in lay eyes was actually the result of the misrepresentation and misunderstanding of a highly complex scientific endeavour. His accusers were wrong in their interpretation of the state of his

\footnotetext{
39 Saundra Saperstein, "Graphic Film Shown at Scientist's Cruelty Trial," The Washington Post, October 28, 1981, C7.

${ }^{40}$ Keith B. Richburg 'Scientist Convicted, Fined \$3,015 for Cruelty to Monkeys', The Washington Post, 24th November 1981, B1.
} 
monkeys because they drew on anthropomorphic analogies with human suffering mixed with comparison to "normal" non-human primate behaviour. Neither reference point was relevant to deafferented monkeys. Much later, Taub was similarly challenged when an interviewer displayed a film showing one of Taub's monkeys propelling himself uncertainly across the floor using a deafferented arm. Taub explained:

[t] hat is not a sad monkey ... A monkey is not a human being; the expressions on a monkey's face are not interpretable in the same way as the expression on the face of a human being. Both Pacheco and Newkirk have had experience with monkeys, and they know that that expression was not sadness. In one of the other photos they always point to, the expression on the monkey's face looks forlorn-if that monkey were a human being, you'd say he was sad-but that expression is not plaintive. That's a monkey before he's going to be fed. He purses out his lips and goes "woo woo woo." ${ }^{41}$

At trial Taub made similar points and consistently emphasised that his monkeys had to be understood in the context of his research programme. It was physiologically impossible to feel anything in deafferented limbs. Physiologically, deafferentation rendered animals incapable of pain. It was nonsense, therefore, to speak of cruelty. Such language revealed the inability of non-specialists to understand and interpret the highly contextualised welfare needs of laboratory animals. Deafferented monkeys could only be properly understood by those with intimate knowledge of both the deafferentation procedure and how an individual monkey has responded to it. In sum, Taub argued that only he could adequately provide for their needs in line with his longstanding culture of care. The consulting veterinarian who sat on the IBR's Animal Care Committee, Paul Hildebrandt, confirmed Taub's claims stating that his training as a pathologist afforded him "little experience with research animals of any sort or with primates in or out of the laboratory." ${ }^{42}$ Clinical veterinarians were not qualified to care for deafferented animals as they had no understanding or experience of their needs or their place within experimental science. Veterinary expertise was suitable in many environments, including the livestock industry, zoological gardens and wider society when dealing with companion animals. However, within the specialist world of Taub's laboratory veterinary knowledge alone was an insufficient resource to adequately understanding the care and welfare needs of deafferented monkeys.

Very few veterinarians had experience with deafferentation but one who did was Adrian Morrison, Professor of Anatomy at the University of Pennsylvania's School of Veterinary Medicine. As witness for Taub's defence, Morrison explained that from a clinical veterinary

\footnotetext{
${ }^{41}$ See Caroline Fraser, "The raid at Silver Spring," The New Yorker, April 19, 1993, 66-84, on 83.

${ }^{42}$ Nancy Henson 'Cruelty to Animals: the state versus the scientist', New Scientist, 3rd December 1981 pp.672-674, p.673.
} 
perspective the most effective treatment for a non-usable limb would be amputation. ${ }^{43}$ Within Taub's laboratory, however, this made no sense given that the desensitised limb was a fundamental part of the experimental system. Initially, District Court Judge Stanley Klavan was sceptical of this testimony. He reminded Morrison of the visual images showing monkeys with open wounds, some with varying states of bandaging, alongside particularly disturbing images of fingers partially or entirely chewed away. How could Morrison, as a veterinarian, account for this evidence which so clearly suggested cruelty? Particularly when other veterinarians had categorically asserted that the animals were suffering. Morrison re-iterated that it was inappropriate to view the animals as though they were human. In Morrison's experienced eye, the Silver Spring Monkeys looked to be in good health. He pointed to the clinical indicators one looked for in laboratory primates such as thick coats of hair, lively eyes, and healthy body weights appropriate to age. ${ }^{44}$ These clinical signs of good care, welfare and health also aligned with more objective evidence. White blood cell counts, for instance, demonstrated the absence of infection. ${ }^{45}$ With desensitized limbs, Morrison further explained, the absence of bandages was a sign of good not bad care. Whereas some monkeys tolerated dressings, others would become irritated and chew through the bandages. Moreover, some wounds benefitted from dressing whilst in other cases an open wound was preferable to risking a bandage becoming so tight it cut circulation. Far from negligence here variation in care evidenced that proper attention had been given to the specific needs of individual animals. Clinical veterinarians who encountered monkeys in zoos, or ethologists observing similar primates in natural settings, were bound to be misled if they brought their understanding to the laboratory without taking into account the artificial nature of these research animals. From the perspective of a deafferented animal the desensitised limb was a curiosity. It was no longer recognised as part of the animal's body. Taub had demonstrated this by showing that when deprived of sight a monkey paid their limb little no attention. However, left to their own devices in normal living spaces, monkeys responded to their senseless limbs in unpredictable ways. They might by ignoring it accidentally damage a desensitized limb. Conversely, becoming overly curious could lead animals to investigate the limb by chewing at digits. Taub recognised that the "tendency toward injury, self-inflicted or otherwise, constitutes one of the major difficulties in carrying out deafferentation experiments with monkeys." ${ }^{46}$ Recognising and overcoming these challenges through adequate care was the fundamental precondition that had made

\footnotetext{
${ }^{43}$ Adrian Morrison, An odyssey with animals: A veterinarian's reflections on the animal rights and welfare debate (Oxford: Oxford University Press, 2009), 21-32.

${ }_{44}$ P.J. Hand and A. R. Morrison, "Veterinary medical issues in the case of Edward Taub," Neuroscience Newsletter 14 (1983): 3.

45 A later "Minority Report" produced by a Public Health Service board of inquiry concluded Taub had provided "good care" for his animals. See E Taub, "Rebuttal to NIH Memorandum," 18, Box 27, file “Taub case \#2," NEM.

${ }^{46}$ Taub, "Movement in nonhuman primates," 343.
} 
Taub's experimental research possible.

On the $23^{\text {rd }}$ November, Klavan ruled that there was no evidence of cruelty in 113 of 119 charges (seven charges for each of the seventeen monkeys). However, Taub was convicted on 6 counts of failing to provide adequate veterinary care. Taub's expertise in the care of deafferented monkeys had been accepted. Yet, he was nonetheless convicted of having failed to provide veterinary care. Why? In part, the ruling reflected the outcome of separate hearings conducted by the NIH who as funder also conducted an inquiry into the allegations against Taub. Following a site visit convened to ensure his procedures complied with animal care guidelines, the NIH suspended support for Taub on October $8^{\text {th }} 1981$ due to his failure to provide suitable veterinary care. Occurring less than twenty days before legal proceedings commenced on the $27^{\text {th }}$ October this was far from an indication of support. However, the NIH was compelled to find a way to take a proactive stance without supporting or condemning Taub. For the NIH the Silver Spring monkey controversy couldn't have occurred at a worse time as congressional hearings were scheduled for the 13th and 14th October to address the acceptable uses of animals within scientific research. Suspending Taub's funding now appears as a forlorn attempt to prevent the hearings focussing on Taub and the Silver Spring monkeys. Taub had been angered by the NIH's decision and was further aggrieved by Klavan's ruling which mirrored it. He therefore appealed choosing this time to stand before a jury on the six remaining counts.

Taub's appellate trial began in June 1982 and was tightly focussed around provision of veterinary care. Following an extensive second trial the jury overturned five of Taub's six convictions endorsing in a sense his expertise and ability to properly care for the animals. The remaining conviction, which involved a monkey named Nero, was upheld due to a pivotal contribution by David M. Renquist of the NIH Veterinary Resources Branch. In October 1981 the Silver Spring monkeys had been placed under Renquist's care following transfer to the NIH animal facility at Poolsville. Shortly after their arrival Renquist and his team concluded that the deafferented arm of a monkey named Nero had to be amputated. During the appellate trial, Renquist explained that the presence of osteomyelitis (a life-threatening infection) indicated a long-standing bone infection that "dated back to a time when the animal was in Dr. Taub's laboratory." ${ }^{47}$ This suggested that Taub had failed to provide adequate veterinary care and at trial appeared to establish this failure had resulted in the need to amputate a limb. ${ }^{48}$ Faced with the unequivocal statement of an NIH veterinarian with first-hand experience of the Silver Spring monkeys the jury upheld the sixth conviction concerning Nero. Nero's story was, however, more complicated than it first appeared. Some months after the ruling a pathology report

47 "Letter from Shelley Steuer concerning Dr. Renquist and the Pathology Report that indicates no Osteomyelitis in Nero's Arm," 2, Box 27, folder "Autopsy report," NEM.

48 "Official Transcript of Proceedings, State of Maryland vs. Edward Taub, 23rd June 1982," 114, Box 27, Folder "Wyngaarden Vs. Taub,” NEM. 
was anonymously passed to Taub revealing that Nero's infection was under control at the time of amputation and there was no evidence of osteomyelitis. ${ }^{49}$ The report was inexplicably absent from the "complete" medical records that had been provided to Taub by the NIH for the purpose of mounting his appeal. Yet both Renquist and the prosecuting assistant state's attorney had access to the information. ${ }^{50}$

The management of Nero illustrates the complexity and confusion that proliferated about how to adequately provide for the care and welfare of deafferented animals. At the NIH, Renquist consulted John Leo Doppman of the Diagnositic Radiology Department on 27 $7^{\text {th }}$ October 1981. Doppman reasoned that Nero's limb should be removed as "human limbs without pain sensation often suffer dislocations or fractures and that repaired joints or fractures are usually reinjured" therefore it was "only a liability to the animal." ${ }^{51}$ Taub, still the legal owner, refused consent believing a more conservative treatment was appropriate emphasising again that the deafferented limb was essential to his experimental programme. Appealing to James Stunkard, a court appointed veterinarian tasked with ensuring the monkeys welfare, led to two NIH neurologists (Drs. Kafta and Sawaya) and an orthopaedic surgeon (Dr. McGown) being consulted. Like Doppman their reference point was human medicine though they reached different conclusions. Given that a human hand would be treated conservatively with debridement and antibiotics due to "the psychological significance of a hand to a human patient" they sided with Taub albeit for different reasons. ${ }^{52}$ For Doppman and the four NIH veterinarians, Nero was conceived as an animal and therefore could be deprived of his limb. For Kafta, Sawaya and McGowan, Nero was treated as a human and so efforts should be made to preserve the limb. For Taub, depriving Nero of his limb was unthinkable. Motivated by a mixture of his intimate knowledge of Nero, his experience of the impact of deafferentation, as well as the necessity of maintaining Nero's integrity if the animal was to continue to contribute to his research programme, Taub ruled out amputation. In the absence of consensus on what was best for Nero no action was taken. From the $29^{\text {th }}$ October to $1^{\text {st }}$ November Nero was treated conservatively. However, by the $1^{\text {st }}$ November a large area of the hand had become necrotic. Taub had been warned that his refusal to consent to amputation was "against the advice of veterinary doctors" and reminded that if Nero was to die "there might be publicity over it" that would be damaging at trial. ${ }^{53}$ Under duress, once necrosis was reported Taub agreed to amputation. It was not until Taub received the leaked pathology report that he

\footnotetext{
${ }^{49}$ M. Morin "Gross Pathological Findings" in Box 27, folder "Autopsy report," NEM.

50 "Letter Shelley Steuer (Attorney Advisor, NIH) to Edgar H. Benner (Arnold \& Porter, Taub's law firm)," 7th February 1983, 2. Box 27, folder "Autopsy report," NEM.

51 "Memorandum, 9th November 1981," Box 27, folder "IBR Monkeys, for the record," NEM.

52 Ibid., 2.

53 "Official Transcript of Proceedings, State of Maryland vs. Edward Taub, 23rd June 1982,” 174, Box 27, Folder "Wyngaarden Vs. Taub," NEM.
} 
learned the amputation was unnecessary. Autopsy of the removed limb revealed that at the time of amputation the infection had been brought under control. Necrotic damage was the result of bandaging applied too tightly cutting off circulation resulting from poor veterinary care at the NIH. Nero had lost an arm not because of events experienced in Taub's laboratory.

Taub's twenty-four years of experience working with deafferented laboratory primates counted for nothing in the face of the perceived authority of veterinary knowledge to determine animal care and welfare. This remained so even when veterinary intervention caused more harm than good. In practice, NIH veterinarians often found "standard veterinary practice" did not provide "entirely satisfactory" care for the Silver Spring monkeys. ${ }^{54}$ On consultation with Taub, they often adopted many of his techniques for caring for deafferented animals. Nevertheless, publically they remained critical and it seemed at least to Taub negative welfare outcomes were routinely ascribed to treatment under his care when often they had been caused by inappropriate veterinary care.

When Taub began his research career in the 1950s the principle scientist was by default the ultimate authority on the care of animals related to their experimental work. It was common for scientists to have their own animal houses managed to their own standards of husbandry and welfare. Centralized animal facilities developed in part as a managerial response to increased external regulation. From the 1960s, animal research developed in the USA was principally governed by two separate but interrelated Federal regimes (in additional to varying state laws). One, enforced by the United Stated Department of Agriculture (USDA) under the 1966 Animal Welfare Act, established standards of care for animals destined for use in scientific research. Another, which evolved within and was enforced by the Public Health Service (PHS), compelled federally funded scientists to commit to standards of practice in the care and welfare of laboratory animals. Both regulatory processes were well established by 1981 though Taub appeared relatively uninformed on either. Taub had been shielded from these significant shifts in regulatory practice as a result of working at a small private research facility. In any case, the 1966 Animal Welfare Act had explicitly stopped short of establishing any regulatory framework for animals during experimentation. Its focus was on the production, provision and transportation of animals that were to be used in experimental science as well as their husbandry and care prior to use. Arthur G. Perry, a USDA veterinarian who under the Animal Welfare Act had inspected Taub's laboratory as recently as the $13^{\text {th }}$ July 1981 explained at Taub's trial that "when you inspect research facilities, there are a number of restrictions that are imposed upon the type of inspection that we can make." ${ }^{55}$ In contrast to inspecting an

\footnotetext{
54 "Major points on which the decision of the HHS grant appeals board contradicts the NIH ad hoc committee findings," 1, Box 27, folder "Wyngaarden vs Taub," NEM.

55 "Witness Dr. Arthur G. Perry transcript 28th June 1982," 17-18. Box 27, folder "Trial de novo 1982 (2)," NEM.
} 
animal breeder or dealer where every aspect of the business could be questioned at a research facility one did not investigate how animals were used during experiment. In any case, Perry's report showed the animals "appeared normal" after a thorough assessment of

their alertness. Their coats. Whether they appeared comfortable ... Whether they are sick, lame, blind, diseased, any of those characteristics. In relation to those animals on that particular day ... I passed that the veterinary care was adequate. ${ }^{56}$

When challenged on his knowledge of regulatory responsibility Taub responded that it was "somebody else's business to be aware of the regulations, not the lab's." ${ }^{77}$ Evidently Taub was not just ill-informed on their nature but considered them to outside the purview of his responsibility. This was an antiquated view at the time though not unusual as animal research regulations had evolved after Taub and many established scientists had begun their research careers. Nevertheless, as far as the USDA was concerned, Taub's laboratory was operating within regulatory expectations.

In contrast to the Animal Welfare Act, PHS regulations engaged more closely with experimental work. A pre-requisite of PHS funding, for example, was to provide written assurance of a commitment to practices as laid out in the Guide for the Care and Use of Laboratory Animals. The PHS regulatory framework operated through a mix of formal and informal expectations governing the relationship of institution to the PHS as opposed to standards enforced by federal law. Carbone has usefully distinguished federal legislation as "top down" having been "thrust upon the scientific community" and the Guide "bottom up" responding to the needs of the research community by enacting standards of care through "flexible self-regulation." 58 Published in 1963, the first edition of the Guide was developed by an independent organisation by veterinarians seeking to improve laboratory animal husbandry known as the Animal Care Panel. Subsequent editions were produced by the National Research Council's Institute of Laboratory Animal Resources. It was revised in 1965, 1968, 1972 and 1978 with each edition placing greater emphasis on veterinary expertise. Importantly, whereas Animal Welfare Act regulations had the status of law those within the Guide (as implied in the name) did not. It communicated best practice assuming an ethos of self-regulation and trust. The Guide was initially advisory but compliance increasingly became expected. Its provisions became authoritative in 1973 when the PHS issued its "Policy on Humane Care and Use of Laboratory Animals" making adherence to the Guide mandatory for federally financed animal research. Subsequently, failure to follow the Guide could lead to suspension of a grant. When the NIH suspended Taub's funding on the grounds that adequate veterinary care as required by the Guide had not been provided it marked a significant change. Henceforth adherence to

\footnotetext{
56 Ibid., 17.

${ }^{57}$ Henson, "Cruelty to animals," 674.

${ }^{58}$ Carbone, What animals want, 38.
} 
the Guide had to be considered a mandatory expectation departure from which had serious consequence (as opposed to an informal set of principles to be adapted to the needs of an experimental programme). ${ }^{59}$ As a result, the independence of principle scientists to interpret the welfare needs of their laboratory animals was partially curtailed in favour of veterinary expertise and the prioritisation of the needs of animals in and of themselves.

For the NIH, failure to provide adequate veterinary care was judged to be serious. So much so that suspension quickly moved to termination. Prior to the site visit to Taub's laboratory the NIH committee had reviewed the evidence against Taub by speaking with local law enforcement and Maryland Assistant State's Attorney. The subsequent report gave considerable weight to the five affidavits collected by Pacheco as well as medical reports produced after the monkeys were taken into police custody by Janis Ott (clinical veterinarian from Brookfield Illinois zoo) and Phillip Robinson (clinical veterinarian from San Diego zoo). Ott and Robinson asserted that "veterinary care available to animals sustaining injuries to deafferented limbs was not sufficient to meet their medical needs and that the medical care in general provided for this colony was inadequate." ${ }^{\text {00 }}$ This appeared to the NIH to confirm nonadherence to the Guide. However, Taub contended that as the then current Guide stated that "[a]dequate veterinary care should be provided by a veterinarian qualified by doctoral training ... or pertinent experience" the apparent absence of veterinary expertise did not equate to an absence of care. ${ }^{61}$ Taub maintained that reference to pertinent experience allowed for scenarios where expertise in specialist animals should stand in the stead of veterinary qualification. Should not his twentyfour years working with nonhuman primates, as well as a unique understanding of the singular nature of deafferented animals, qualify as pertinent experience to provide adequate veterinary care? Taub's reading reflected a much earlier performative ethos that had shaped the Guide where a degree of latitude existed as to how general principles and standards were best enacted at the institutional level. The NIH, however, took a more literal position in claiming that a qualified veterinary professional was required. This led to the termination of his grants and effectively ended Taub's career in animal research.

In February 1984, a Health and Human Services (HHS) Departmental Grant Appeals Board favoured Taub's interpretation noting that:

after many years of working with primates the PI [Principal Investigator] probably had acquired considerable general knowledge about the animals and their care ... although the $\mathrm{NIH}$ veterinarians criticized the PI's treatment of certain problems, they adopted some of the PI's techniques, after consulting with the PI on several occasions while the monkeys were in their custody, and that their own

59 The use of animals, 76-93.

60 Ibid., 83.

${ }^{61}$ ILAR, Guide for the care and use of laboratory animals (Bethesda: MD: Public Health Service, 1978), 11. 
early treatment, based on standard veterinary practice, did not prove entirely satisfactory. ${ }^{62}$

The HHS appeals board also made clear that "no evidence monkeys were harmed by lack of veterinary supervision, or that the condition of the monkeys showed inadequate veterinary care" - an observation that had been lost during the original NIH investigation. ${ }^{63}$ For Taub this was a pyrrhic victory. The HHS appeals board endorsed Taub's ability to care for the monkeys and found no indication the animals had suffered unduly. However, it nevertheless upheld the NIH's right to terminate Taub's grant. In August of the previous year the Court of Appeals of Maryland had reversed the single remaining conviction of having failed to provide veterinary care for Nero on the grounds that the Maryland Code did not apply to medical research. By 1984 Taub had cleared his name but this did very little to restore his reputation. Nor did it result in the restoration of NIH support for his research.

In 1981 Taub was caught out of step with wider shifts in the culture of laboratory animal care as well as public expectations of those engaged in animal research. Taub's argument that by virtue of his experience he was uniquely and better qualified than clinical veterinarians to care for deafferented animals proved unconvincing in large part to due to political necessity. ${ }^{64}$ The NIH had also been caught off guard with upcoming congressional hearings addressing animal research it was eager to be seen as "a proper steward of public funds." ${ }^{65}$ In seeking to distance itself from Taub, without explicitly supporting the accusations of cruelty against him, the NIH unintentionally located itself closer to his critics than his supporters. David Rioch, chair of the IBR's Animal Care Committee, believed that the NIH was too eager to follow the lead of veterinarians sympathetic to animal advocacy politics who were "applying human expectations of pain to animal surgery [which was] inappropriate because pain is primarily a matter of societal conditioning to which animals are not subject." 66 The inelegant response of the NIH repeatedly erred in favour of what we might call the humanitarian veterinary perspective. In the face of evidence that the absence of professional veterinary expertise had had no impact on the welfare of animals and that its introduction in several instances caused harms the NIH nevertheless prioritised veterinary authority when it came to laboratory animal care.

\footnotetext{
62 "Major points on which the decision of the HHS grant appeals board contradicts the NIH ad hoc committee," 1, Box 27 folder "Wyngaarden vs Taub," NEM.

63 "Appeals Board Decision" quoted in letter James B. Wyngaarden Neal E. Miller, 2nd June 1986, p.2. Box 27, folder "Wyngaarden vs Taub," NEM.

64 "Memorandum in support of the respondents position," 15. Box 27, folder "Taub case\#2 Trial de novo, 1982," NEM.

${ }^{65}$ Letter J. B. Wyngaarden to N. Miller, 27th December 1983, Box 27, folder "Taub Case, 1983," NEM.

66 "Report and Recommendations of the NIH Committee to Investigate Alleged Animal Care Violations at the Institute for Behavioural Research, 5th October 1981," p. 13. Box 27, folder “Taub case \#2 trial de novo," NEM.
} 
In doing so, the NIH mobilised similar forms of veterinary expertise that had been set against Taub by animal advocacy groups. In contrast, veterinarians such as the USDA inspector, Perry, were relatively educated in, and willing to accommodate the needs of, experimental research within their estimation of appropriate care and welfare. In cross examination, Perry revealed he had developed an interest in Taub's deafferentation research as it stood out from more routine toxicity testing and appeared to be "of real historical significance in the field of medicine." ${ }^{37} \mathrm{In}$ contrast, Janis Ott and Phillip Robinson were veterinarians familiar with primates, respectively of Brookfield (Illinois) and San Diego zoos. Both had been drawn into the Silver Spring monkey episode by PETA. They conducted examinations with little to no familiarity with Taub's scientific research and in the absence of any knowledge of deafferented animals. In justifying the decision to suspend and later terminate Taub's grants the NIH committee put on record that Taub's "lack of care is further evidenced by the examination report prepared by Drs Ott and Robinson" thereby validating the argument against Taub. ${ }^{68}$ The NIH failed to distinguish between veterinarians who had long worked within the animal research community and had become accustomed to its practices and those whose expertise was based on experience of entirely different contexts.

Taking seriously the normative standpoints of veterinarians working in zoos and ethologists observing animals in nature meant prioritising the needs of the animals as a member of its species over the needs of an experimental system. Ott and Robinson interpreted the experience of the Silver Spring monkeys through reference to their experience of captive animals in zoos, knowledge of non-human behaviour in nature and loose anthropomorphic reasoning. So too did Michael W. Fox, Donald Barnes, Geza Teleki, John McArdle and Ronnie Hawkin in the original affidavits that had been passed to the local law enforcement. In taking these interpretations seriously, arguably more seriously than USDA veterinarians and Taub himself, the NIH inadvertently gave credibility to a much broader culture of care than had previously been the norm even within laboratories that had fully integrated veterinary care into their practice. The difference was stark. Animals could now suffer in the absence of physiological pain. Whether or not an animal was physiologically capable of feeling pain, one had to consider the behavioural, mental and social needs of the species. As such, the NIH unintentionally empowered an alternative and to some degree radical culture of animal care which subsequently took centre stage triggering the reform of the Animal Welfare Act 1966.

In October 1981 the Subcommittee on Science, Research and Technology of the US House of Representatives devoted considerable time to discussing the ongoing Silver Spring monkey

67 "Witness Dr. Arthur G. Perry transcript 28th June 1982," pp. 17-18. Box 27, Folder "Trial de novo" 1982 (2), NEM.

${ }^{68}$ Report on the Allegations of Noncompliance with Public Health Service Policy Governing the Care of Laboratory Animals at the IBR, 7th Oct 1981, p. 8. Box 27, Folder “Taub Case - Trial \#1," NEM. 
controversy. The hearing focussed on "the proper balance between freedom of inquiry in medical research and the suffering of animals used in experiment." ${ }^{69}$ Taub was not present but his work and the Silver Spring monkeys were the basis of evidence provided by Alex Pacheco and Michael Fox as well as William F. Raub, Joe R. Held and other NIH representatives. In his testimony, Fox developed a clear and particularly influential argument as to why a much broader culture of care was required within American laboratories. Fox, a British trained veterinarian with a $\mathrm{PhD}$ in ethology, had moved to the USA in 1964 to pursue a research career. Having held a number of academic positions studying the psychology and behaviour of animals he gradually shifted his career from animal research to animal advocacy. In 1976 he became director of the Institute for the Study of Animal Problems (Washington, D.C.) and later Scientific Advisor to the Humane Society of the United States. Fox had studied how psychological stress had physiological consequence during his scientific career. He now argued that mental wellbeing had to be taken seriously by animal researchers as psychic or physiological distress would corrupt experimental data. The social and mental needs of laboratory animals had to be provided for and could be understood in the context of the natural and evolutionary history of the species, ethological knowledge and properly deployed anthropomorphism..$^{70}$ In his prepared affidavit Fox explained that:

Monkeys are highly complex, social animals with an emotional system much like our own. It is my professional opinion that the monkeys I viewed at the IBR on August 28, 1981 were, without exception, suffering unnecessarily from various causes, including physical and psychological deprivation, a lack of veterinary care and a failure to provide proper, basic environmental needs. ${ }^{71}$

Fox went on to emphasise that the problem was more than the fact that Taub had failed to meet the expected animal welfare standards of the day. The critical issue was that Taub had underestimated the degree to which failure to provide for the natural, social, mental and behavioural needs of the Silver Spring monkeys would have corrupted his experimental data. Fox outlined a broad culture of care that made scientific knowledge dependent on the promotion of animal welfare in the widest sense. Left unsaid, but implicit to the congressional inquiry, was that millions of federal dollars had been funnelled to Taub and others via the $\mathrm{NIH}$ and if Fox was to be believed the scientific data produced must be judged as less than reliable. $^{72}$

\footnotetext{
${ }^{69}$ The use of animals, 1.

${ }^{70}$ For his defence of anthropomorphism see Michael Fox, Laboratory Animal Husbandry: Ethology, Welfare, and Experimental Variables (Albany, NY: SUNY Press, 1986), 182.

${ }^{71}$ The use of animals, 48.

72 The animal rights advocate Henry Spira gave more explicit testimony on economic implications asserting "this subcommittee has an almost unique opportunity of doing what is good for the public, for the lab animals, and for the productive science while saving taxpayers' dollars" (The use of animals, 277).
} 
Fox's argument was distinct from historical antivivisectionist criticism which the congressional hearing would have been more than familiar with. Fox was not presenting a moral argument against the corruptive evils of cruelty to animals. Instead, Fox made the needs of experimental science dependent on those of animal welfare advocating for a culture of care where all aspects of animal wellbeing served as guarantors of reliable science. Moreover, he mobilised science to argue for legislative reform. The 1966 Animal Welfare Act had established "provisions for the physical requirements of laboratory animals" yet recent advances in ethology and laboratory animal science had shown that:

Deprivation of social and environmental needs of primates and of other laboratory animals housed in cages often in social isolation and without sufficient freed of movement can be as bad in terms of the animal's welfare and the validity of experimentation as depriving it of adequate nutrition. ${ }^{73}$

Safeguarding animal welfare was "an ethical imperative as well as a scientific imperative because animals that are not optimally cared for will jeopardize scientific progress." ${ }^{74}$ Even if Taub's monkeys could not feel physiological pain their self-mutilation was an indicator of stress. But it was equally a signal that cast doubt on the quality and validity of Taub's data. Fox and others had been advocating for the co-dependency of the quality of science and the promotion of animal welfare for over a decade. The Silver Spring monkeys provided a platform which, through virtue of its political and public character, brought the argument centre stage.

\section{Conclusions}

In the immediate years following the 1981 raid on his laboratory Taub endured three trials and an NIH investigation before clearing his name in 1984. He never returned to animal research. Instead, he embarked on what in many ways became an equally if not more successful research trajectory. Joining the University of Alabama-Birmingham in 1987 he applied what he learned with monkeys to the development of new treatments for human stroke victims. Taub revitalised approaches to rehabilitation after brain injury by demonstrating that what had been thought of as a physiological limit was in fact "learned non-use." 75 Clinical approaches to rehabilitation following brain injury assumed a fixed limit beyond which patients could regain no movement. This view derived from the belief in a static brain unable to adapt to damage. Working with monkeys had taught Taub that the brain was far more flexible than many believed. Having seen similar behaviours in his monkeys Taub was convinced that human patients simply gave up

\footnotetext{
73 Ibid., 186

${ }^{74}$ Ibid., 189.

75 E. Taub, G. Uswatte, and R. Pidikiti, "Constraint-Induced Movement Therapy: a new family of techniques with broad application to physical rehabilitation--a clinical review," Journal of Rehabilitation Research and Development 36, no. 3 (1999): 237-51.
} 
the hard work of retraining the brain to use an affected limb. It was easier to compensate with the unaffected limb. Adapting Taub's experimental techniques to human patients involved constraining healthy limbs by force so as to compel consenting individuals to learn anew how to move nonresponsive limbs. The outcome, Constraint-Induced Movement Therapy, revealed the fallacy of existing approaches to rehabilitation as patients found they could relearn movement beyond the presumed physiological limit.

Constraint-Induced Movement Therapy was a powerful clinical application demonstrating a radical new understanding of the brain: neuroplasticity. The Silver Spring monkeys' final contribution to science was to provide substantive evidence base for the theory. By 1987 the controversy had evolved from arguments over what had been done to the animals in the name of science to the equally vexed question of what was to be done with them in the future. On the one side, scientific organisations including the Society for Neuroscience and the American Physiological Society had offered to raise an endowment to cover the cost of maintaining the animals for their remaining natural life spans. On the other, a coalition of animal advocacy groups, including PETA and led by Congress Representative Robert C. Smith, wished to purchase the animals in order to guarantee their freedom from science. In congress, 229 members co-sponsored a bill which, had it passed, would have ensured the Silver Springs monkeys be placed in a private animal sanctuary. An independent legal argument went all the way to the Supreme Court which ruled that animal advocacy groups' possessed no legal standing to intervene in the fate of the monkeys. As the debate over the future of the monkeys raged the NIH was underwriting the not insubstantial cost of maintaining the animals now temporarily relocated to the Delta Regional Primate Center in Louisiana. Eventually, the NIH negotiated a compromise where four of the healthier monkeys were rehomed at the San Diego Zoo whilst those deemed to be experiencing unacceptable suffering were euthanized and studied to ensure their deaths would not be in vain. The decision to euthanize was highly controversial. Congressman Smith accused the NIH of violating a commitment to conduct no further research on the animals. Pacheco claimed the NIH killed the animals because "they don't want animals out there who lived to tell their story - they don't want survivors." ${ }^{\prime 6}$ Yet, the decision to euthanize and study those animals deemed unable to adapt to life in the zoo revealed a significant unintended consequence of the long-running controversy. The seizure and subsequent legal wrangling over the fate of the Silver Spring monkeys had prolonging the lifespan of unique animals whose brains had been deprived of sensory stimulation for an unprecedented length of time. Far longer than otherwise would have been the case. ${ }^{77}$ When euthanized and studied in 1991 their brains showed widespread cortical reorganisation

\footnotetext{
${ }^{76}$ Robert Reinhold, "Fate of Monkeys, Deformed for Science, Causes Human Hurt After 6 Years," New York Times May 23, 1987, 8.

77 Joseph Palca, “Famous monkeys provide surprising results,” Science 252, no. 5014 (1991): 1789.
} 
providing the first significant evidence of the hitherto unknown scale of neuroplasticity. ${ }^{78}$ In this way, the Silver Spring controversy had serendipitously enhanced the value and contribution of the monkeys to science.

Retrospectively, the Society for Neuroscience and other scientific organisations celebrated Taub's work as an exemplar of "translational" medicine and evidence of the centrality of animal research to clinical innovation. ${ }^{79}$ This was a strategic rebuttal of critiques by organisations such as PETA. Recasting history in this way erased any recognition that Constraint Induced Movement Therapy and the related significant evidence for neuroplasticity were unintended and serendipitous consequences of criticisms of animal research. In general, media reportage performed a similar reduction by portraying the Silver Spring monkey controversy as a conflict between experimental science and the rights of animals. However, a reflective New Scientist editorial from 1981 presented a more nuanced observation:

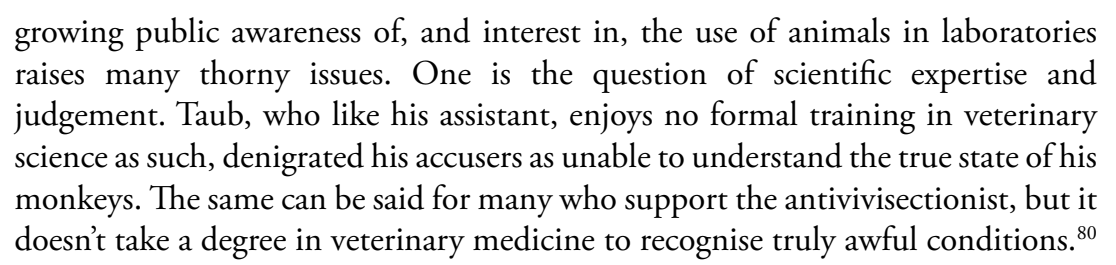

Here we see that specialist expertise was no longer the singular test of authority in determining standards of animal welfare in the laboratory. In a democratic society, public interest in the scientific uses of animals had imbued common sense lay understanding with a credibility of its own. In testimony to the 1981 congressional hearings, Christine Stevens of the Animal Welfare Institute made this point explicit by demanding the mandatory inclusion of public representatives on institutional animal research oversight committees as well as the NIH decision making process for research grants. ${ }^{81}$ Like the previously considered arguments for the relevance of veterinary, ethological and other specialisms, the argument for the inclusion of lay members of the public reflects a culture of care which seeks to increase rather than decrease plurality of perspectives on what should and should not form a matter of concern to laboratory animal welfare. Such an open and inclusive culture of care reflects Puig de la Bellacasa's observation that "to effectively care for a thing we cannot cut off from the composition of its political ecology those we disagree with but who are nevertheless concerned by the thing and

${ }_{78}$ T P. Pons, P. E. Garraghty, A. K. Ommaya, Jon H. Kaas, E. Taub, and M. Mishkin, "Massive Cortical Reorganization After Sensory Deafferentation in Adult Macaques," Science 252, no. 5014 (1991):185760 .

79 Society for Neuroscience, Translational Neuroscience Accomplishments (n.d.): 5.

80 "The unacceptable side of animal research," New Scientist 92, no. 1282 (December 3, 1981): 646.

81 The use of animals, 199. 
the issues it brings to matter." ${ }^{\prime 2}$ Indeed, for Puig de la Bellacasa a condition of caring well is to work to cultivate collective and accountable knowledge without closing the space for dissent. ${ }^{83}$

The Silver Spring monkey controversy gave voice to a constellation of concerns, shaped by veterinary medicine, quasi-humanitarian values and embodied in roles and professions which were distinct to and some way outside of situated research and scientific programmes. It remade the fabric of the culture of care for laboratory animals in late twentieth-century America. Following extensive congressional debate the Health Research Extension Act (1985) empowered the NIH to revise the Public Health Service (PHS) Policy on Humane Care and Use of Laboratory Animals. Subsequent regulations placed greater emphasis on the mental, emotional and social wellbeing of research animals. ${ }^{84}$ Similar changes were made in a revision of the 1966 Animal Welfare Act. The new regulative frameworks established a federal expectation that institutions should establish animal care committees whose membership must include at least one doctor or veterinarian and an individual who has no association with the institution..$^{85}$ Both the revised Animal Welfare Act and the Health Research Extension Act allowed the non-affiliated member to possess scientific expertise. However, the 1986 Public Health Service Policy introduced the need to include an individual "whose primary concerns are in a nonscientific area (for example, ethicist, lawyer, member of the clergy)." ${ }^{\prime 6}$ Bringing perspectives together in this way constituted a culture of care that held differences in opinion within a productive tension with the aim of facilitating meaningful change. Far from an example of the polarization of science from society, the case of the Silver Spring monkeys illustrates the need to find ways to include and thus diversify the forms of concern which shape cultures of care within animal research.

\section{Acknowledgements}

A version of this article was first produced for Humans and Animals at the Margins: An international workshop organised by Mette N. Svendsen and Lene Koch at the The Royal Danish Academy of Sciences and Letters, Copenhagen, Denmark, August 25-27, 2016. I'm grateful to Mette, Lene, and the participants of this meeting for their enthusiastic engagement and subsequent contributions to the development of this research as well as their generous

\footnotetext{
82 Puig De la Bellacasa, Matters of Care, 44-5.

${ }^{83}$ Maria Puig de la Bellacasa, "Nothing comes without its world': thinking with care," Sociological Review 60, no. 2 (2012): 197-216, on 205.

${ }^{84}$ ILAR, Guide, 12-13; Health Research Extension Act of 1985 Public Law 99-158, November 20, 1985, „Animals In Research“; T. L. Wolfle, “Psychological Well-Being of Nonhuman Primates: A Brief History," Journal of Applied Animal Welfare Science 2, no. 4 (1999): 297-302.

85 Public Law 99-158, "Health Research Extension Act (1985)," section 495.

${ }^{86}$ Public Health Service, Public Health Service Policy on the Humane Care and Use of Laboratory Animals, Washington DC US Department of Health and Human Services (Code of Federal Regulations, 1986).
} 
willingness to welcome a nomadic historian into their anthropological world and teach him Danish hygge. This article has also benefitted from guidance and criticism offered by anonymous referees and the editors for which I am thankful.

\section{Competing interests}

The author has declared that no competing interests exist.

\section{Funding}

This work was supported by the Wellcome Trust [grant number 106639]. 